\title{
A Study on Morbidity Management among Lymphatic Filariasis Patients in Udupi district, Karnataka, India
}

\author{
Kataraki Basavaraj, ${ }^{\text {a }}$ Shetty K Bharatesh, ${ }^{2 b}$ Dhar Murali, ${ }^{3 c}$ Kamath Ramachandra, ${ }^{4 a}$ Malamardi Sowmya ${ }^{5 a}$
}

\section{Kataraki Basavaraj, ${ }^{\text {1a }}$ Shetty K Bharatesh, ${ }^{2 b}$ Dhar Murali, ${ }^{3 c}$ Kamath Ramachandra, ${ }^{4 a}$ Mala- mardi Sowmya ${ }^{5 a}$}

'Current Affiliation: Regional Coordinator - Karnataka, DNRT, National AIDS Control Organisation, Ministry of Health and Family Welfare, Govt. of India. ${ }^{a}$ Affiliation where the work was primarily carried out: Dept. of Public Health, Manipal University, Manipal, Karnataka, India.

${ }^{2}$ Current Affiliation: Additional Project Director, Karnataka Health Promotion Trust, Bengaluru, India.

${ }^{b}$ Affiliation where the work was primarily carried out: Lecturer, Melaka Manipal Medical College, Manipal University, Manipal, Karnataka, India.

${ }^{3}$ Current Affiliation: Associate Professor, Department of Population Policies \& Programmes, International Institute for Population Sciences, Govandi Station Road, Deonar, Mumbai - 400088, India 'Affiliation where the work was primarily carried out: Associate Professor, Department of Statistics, Manipal University, Manipal, Karnataka, India. ${ }^{4}$ Current Affiliation: Associate Professor, Dept. of Community Medicine, Melaka Manipal Medical College, Manipal University, Manipal, Karnataka, India. ${ }^{a}$ Affiliation where the work was primarily carried out: Head of Dept, Dept. of Public Health, Manipal University, Manipal, Karnataka, India.

${ }^{5}$ Current Affiliation: Senior Project Consultant, Ernst \& Young, Gurgaon, India.

${ }^{a}$ Affiliation where the work was primarily carried out: Dept. of Public Health, Manipal University, Manipal, Karnataka, India.

'Current Affiliation: Regional Coordinator - Karnataka, DNRT, National AIDS Control Organisation, Ministry of Health and Family Welfare, Govt. of India.

\section{Correspondence}

Dr Basavaraj Kataraki

1872, 22nd A Main, Sector 2, HSR Lay-

out, Bengaluru, Karnataka, India

Mobile no: 9632601819

Email: basavraj.katarki@gmail.com

\section{History}

- Submission Date: 15-09-16

- Revised Date: 07-03-17

- Accepted Date: 17-03-17

DOI : 10.5530/ijmedph.2017.2.18

Article Available online

http://www.ijmedph.org/v7/i2

\section{Copyright}

(C) 2017 Phcog.Net. This is an openaccess article distributed under the terms of the Creative Commons Attribution 4.0 International license.

\begin{abstract}
Context: Morbidity management is one of the key aspects in the life of Lymphatic Filariasis (LF) patients. National Vector Borne Disease Control Programme (NVBDCP) is giving more emphasis by conducting morbidity management training camps every year since 2004 to help LF patients to manage and cope-up with the existing morbidity. Aim: To study the morbidity management among LF patients in Udupi district, Karnataka. Study Setting and Design: The study was conducted in Udupi district, Karnataka, India in 2013. The design was stratified random sampling with proportional allocation method. Materials and Methods: Community based cross sectional study, 178 subjects suffering from LF were selected using stratified random sampling with proportional allocation method. An interview based method was used to collect the data. Lympoedema Morbidity Management Questionnaire were designed based on the guidelines provided by National Vector Borne Disease Control Programme (NVBDCP). Results: Among 178 LF patients only about 34\% had knowledge about the disease causation. About $98.3 \%$ had good hygiene practice towards affected area but only $30 \%$ of them regularly practiced one or the other exercise as recommended. Conclusion: The study clearly indicates the outreach focus on LF patients needs to be enhanced by grass root level health workers. The program has to ensure that all the LF patients will undergo morbidity management training and know how to take care of lymphoedema.

Key words: Morbidity Management, Lymphatic Filariasis, Mass Drug Administration.
\end{abstract}

\section{INTRODUCTION}

Lymphatic filariasis (LF), a mosquito-borne disease, is caused by the parasitic filarial nematodes (roundworms) Wuchereria bancrofti (W. bancrofti), Brugia malayi (B. malayi), or Brugia timori (B. timori). Because the burden of the disease is determined by the intensity and the duration of the infection, the greatest impact of LF is on older age groups. ${ }^{1} \mathrm{LF}$ is one the Neglected Tropical Diseases (NTDs). In 1997 World Health Organisation (WHO) declared LF as eradicable or potentially eradicable and in 2000 the Global Programme to Eliminate LF was launched. ${ }^{2}$ Mass Drug Administration as one of the key strategy to eliminate LF and disability management is the other key component. The programme has a coverage of more than 500 million implemented in 27 countries. $^{3}$ According to World Health Organization (2011) an estimated 120 million people in 73 countries are currently infected with filariasis. It is one of the second leading cause of disability worldwide. ${ }^{4}$ About 15 million people are having lymphoedema (Elephantiasis) and 25 million with urogenital swelling especially the scrotal hydrocele. Around $65 \%$ of those at risk reside in WHO's South East Asia Region. India, Indonesia,
Nigeria, and Bangladesh contribute about $70 \%$ of infection worldwide. ${ }^{5}$

LF is a disfiguring and disabling disease which is usually acquired in childhood and manifestations are usually seen in adulthood. Lymphoedema is common with frequent attacks of infection causing swollen limbs, severe pain and fever. The disease is mainly transmitted by mosquito species Culex quinquefasciatus, Aedes aegypti and Mansonia annuliferal M. uniformis. ${ }^{4,5,6}$ Culex species is main vector for $W$. Bancrofti and Mansonia is the vector for B. Malayi. Most of the time the disease is asymptomatic and the individual may carry millions of larval parasites called microfilariae in the blood and adult worms mainly in the lymphatic system. ${ }^{7}$ These adult worms get blocked in lymphatic system causing lymphoedema like lymphoedema of legs, scortum, breasts and arms. ${ }^{8}$ In India about 600 million people are considered at risk of developing the disease like LF. Indigenous LF is reported from 20 states including the union territories. An average of 250 districts have been identified to be endemic for filariasis. In India the filariasis is mainly caused by two species Wuchereria bancrofti and Brugia malayi. It is estimated
Cite this article : Basavaraj K, Bharatesh SK, Murali D, Ramachandra K, Sowmya M. A Study on Morbidity Management among Lymphatic Filariasis Patients in Udupi district, Karnataka, India. Int J Med. Public Health. 2017; 7(2):9296. 
that $99.4 \%$ of the cases are caused by Wuchereria bancrofti and $0.6 \%$ by Brugia malayi. ${ }^{9}$ In Karnataka there are 8 endemic districts for filariasis and Udupi is one among them. The main aim was to study the morbidity management among LF patients and the objective was to know the knowledge and practices for morbidity management of lymphoedema.

\section{MATERIALS AND METHODS}

A community based cross-sectional study was conducted in Udupi district which is administratively divided into three taluks, namely Udupi, Kundapura, and Karkala, between the months February and July 2013. All the subjects confirmed positive for LF were included in the study and who are bedridden and unable to speak were excluded from the study. Among the total 800 LF patients (District Vector Borne Disease Control (DVBDC), Udupi, 2012) in Udupi district, a sample of 224 patients was included in the study and out of which 178 agreed to participate in the study. A stratified random sampling with proportional allocation technique was used as sampling technique. Number of patients needed for the study, were selected according to urban and rural setting. Then proportionally allocated to all the three talukas (Udupi, Karkala and Kundapur) by randomizing the list of participants from the line list of LF patients given in the Udupi District (DVBDC, Udupi, 2012) in which name and addresses of the patients are mentioned. The randomly selected patients were included in the study. The sample size of 224 for the study was calculated based on the prevalence study which was found in the literature review and calculated using Prevalence $(P)=30 \%$, Confidence Interval of $95 \%$, Relative precision $(\varepsilon)=20 \%$ of p. SPSS version 15 (SPSS Inc., Chicago, IL) was used to analyse the data. Statistical analysis was done with categorical variables expressed as frequencies and percentages. Continuous variables were measured using mean and standard deviation.

\section{Ethical approval}

The ethical clearance was obtained from the Institutional Ethics Committee of Kasturba Hospital, Manipal Univesity; and measures were un- dertaken to maintain patient confidentiality throughout the study and during analysis of data. Written informed consent was obtained from each participant.

\section{Tools}

Included the socio-demographic details followed by lympoedema morbidity management questionnaire ${ }^{10}$ which was designed based on the guidelines provided by NVBDCP for morbidity management among lymphatic filariasis patients. This tool included knowledge about nail hygiene, washing of the affected part, exercises as recommended.

\section{RESULTS}

A total of 178 participants were covered in the study. Majority of the participants were from Udupi taluk (67.4\%) and among the three talukas, maximum participants were from rural (78\%) (Table 1). In this study majority of the participants were females patients (66.3\%), $88.8 \%$ were Hindu and close to $61 \%$ were above 56 years, $75 \%$ male and $52 \%$ female respondents were married. About $30 \%$ of male and $31 \%$ of female respondents were educated up to primary and $15 \%$ of male and $34 \%$ of female were illiterate. Regarding occupation $73 \%$ female were housewives and among men $28 \%$ were unskilled workers (Table 2).

Grading of lymphoedema showed that $52.2 \%$ had grade I staging, 31\% had grade II staging and $13 \%$ had grade III (elephantiasis) staging. Single leg morbidity was high among the respondents, 33\% men had lymphoedema of left leg, and $25 \%$ had on right leg. Among women $44.07 \%$ had lymphoedema of left leg and $37.29 \%$ of right leg. In men $33 \%$ had scrotal swelling (Hydrocele). Knowledge of how the disease is caused was responded by $34 \%$. Women (32\%) were more aware about the disease than men (22\%). For morbidity management, $58 \%$ were aware about nail hygiene and $94.4 \%$ had the knowledge of Mass Drug Administration (MDA). Regarding recommended practices for morbidity management, 98\% patients said they wash the affected body part daily with soap. Regarding frequency of cleaning affected part, $62 \%$ wash twice daily and $17 \%$ wash during morning and evening time. Among 178 patients inter-

Table 1: Distribution of cases according to Taluks.
\begin{tabular}{cccc} 
Taluks & Urban $\mathbf{n}(\%)$ & Rural $\mathbf{n}(\%)$ & Total (\%) \\
Udupi & $21(11.79)$ & $99(55.62)$ & $120(67.4)$ \\
Kundapur & $12(6.74)$ & $26(14.61)$ & $38(21.35)$ \\
Karkala & $6(3.37)$ & $14(7.87)$ & $20(11.24)$ \\
Total & $39(21.9)$ & $139(78)$ & 178 \\
\hline
\end{tabular}

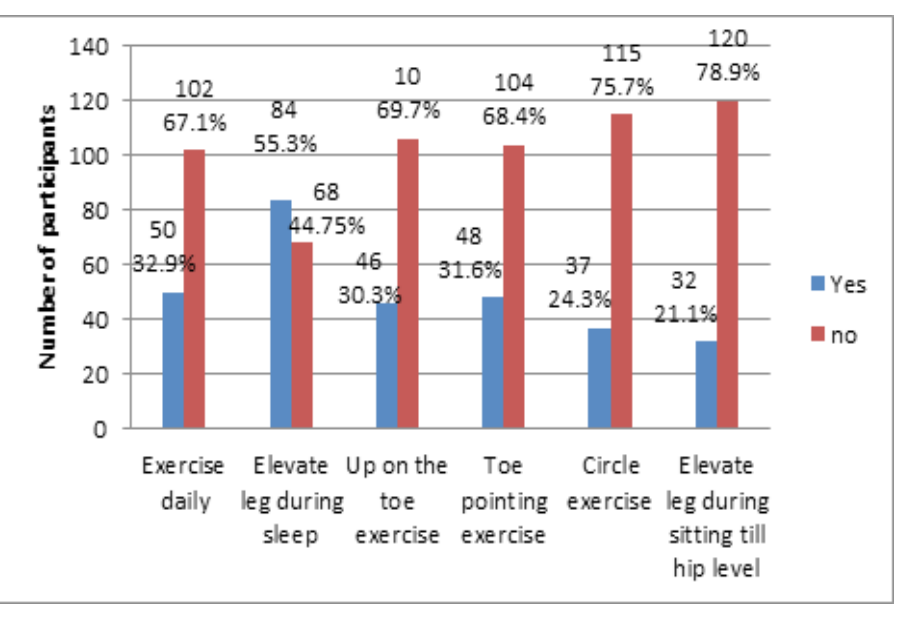

Figure 1: Exercises practiced for morbidity management $(n=152)$

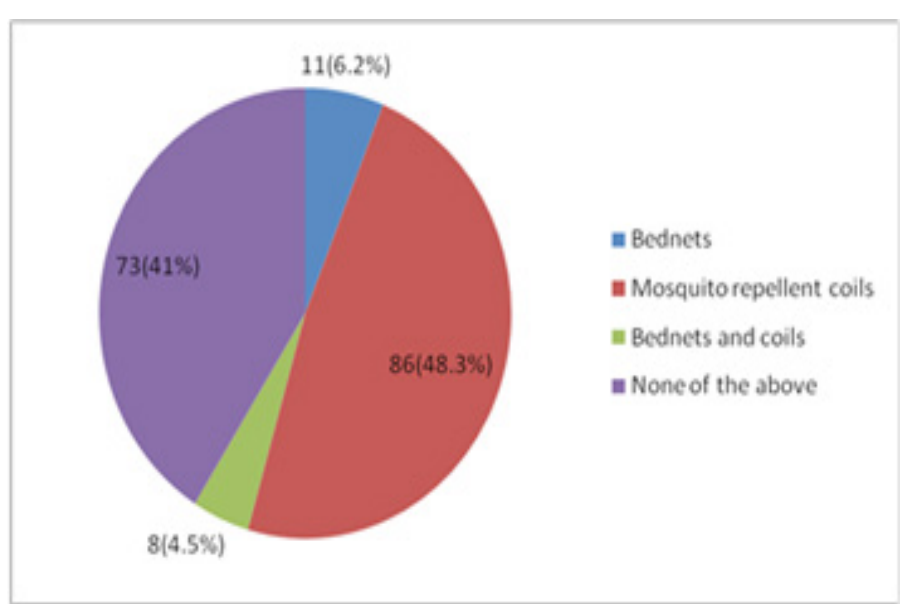

Figure 2: Protective Measures Used by Patients 


\begin{tabular}{|c|c|c|c|c|c|}
\hline \multicolumn{2}{|c|}{$\mathrm{N}=178$} & \multirow{2}{*}{$\begin{array}{c}\text { Male } \\
\mathrm{N}\end{array}$} & \multicolumn{3}{|c|}{ Female } \\
\hline Variables & Category & & $\%$ & $\mathrm{~N}$ & $\%$ \\
\hline Gender & & 60 & 33.7 & 118 & 66.3 \\
\hline \multirow[t]{3}{*}{ Religion } & Hindu & 55 & 91.7 & 103 & 87.3 \\
\hline & Muslim & 3 & 5.0 & 10 & 8.5 \\
\hline & Christian & 2 & 3.3 & 5 & 4.2 \\
\hline \multirow[t]{6}{*}{ Age (yrs) } & $<15$ & 0 & 0 & 1 & 0.8 \\
\hline & 16 to 25 & 2 & 3.3 & 1 & 0.8 \\
\hline & 26 to 35 & 2 & 3.3 & 2 & 1.7 \\
\hline & 36 to 45 & 5 & 8.3 & 15 & 12.7 \\
\hline & 46 to 55 & 15 & 25.0 & 27 & 22.9 \\
\hline & $>56$ & 36 & 60.0 & 72 & 61.0 \\
\hline \multirow[t]{5}{*}{ Marital status } & Married & 45 & 75 & 62 & 52.5 \\
\hline & Unmarried & 10 & 16.7 & 10 & 8.5 \\
\hline & Widow & & & 44 & 37.3 \\
\hline & Widower & 5 & 8.3 & & \\
\hline & Single & & & 2 & 1.7 \\
\hline \multirow[t]{7}{*}{ Educational status } & Primary & 18 & 30.0 & 37 & 31.4 \\
\hline & Higher primary & 22 & 36.7 & 26 & 22.0 \\
\hline & High school & 10 & 16.7 & 11 & 9.3 \\
\hline & Pre University & 0 & 0 & 2 & 1.7 \\
\hline & Graduate & 1 & 1.7 & 1 & 0.8 \\
\hline & Post graduate & 0 & 0.0 & 0 & 0.0 \\
\hline & Illiterate & 9 & 15.0 & 41 & 34.7 \\
\hline \multirow[t]{8}{*}{ Occupational status } & Professional/ white collar & 1 & 1.7 & 2 & 1.7 \\
\hline & Business & 9 & 15.0 & 2 & 1.7 \\
\hline & Skilled & 10 & 16.7 & 1 & 0.8 \\
\hline & Unskilled & 17 & 28.4 & 18 & 15.3 \\
\hline & Housewife & & & 88 & 73.3 \\
\hline & Retired & 6 & 10.3 & 2 & 1.7 \\
\hline & Right now not working & 12 & 20.7 & 1 & 0.8 \\
\hline & Unemployed & 3 & 5.2 & 6 & 5.0 \\
\hline
\end{tabular}

\begin{tabular}{|c|c|c|c|}
\hline Characteristic & $\mathrm{N}(\%)$ & Characteristic & N (\%) \\
\hline \multicolumn{4}{|c|}{ Knowledge } \\
\hline How the disease is caused & & About MDA & \\
\hline Yes & $60(33.7)$ & Yes & $168(94.4)$ \\
\hline No & $118(66.7)$ & No & $10(5.6)$ \\
\hline \multicolumn{4}{|l|}{ Nail Hygiene } \\
\hline Yes & $102(57.3)$ & & \\
\hline No & $76(42.7)$ & & \\
\hline \multicolumn{4}{|c|}{ Morbidity management } \\
\hline Sites affected & Males $n=60$ & & Females $n=118$ \\
\hline Rt arm & $1(1.6)$ & & $3(2.5)$ \\
\hline Lt arm & $2(3.3)$ & & $1(0.8)$ \\
\hline Rt Leg & $15(25)$ & & $44(36.7)$ \\
\hline
\end{tabular}




\begin{tabular}{|c|c|c|c|}
\hline Lt leg & $20(33.3)$ & & $54(45.0)$ \\
\hline Both Legs & $3(5.2)$ & & $18(15.0)$ \\
\hline Scortum & 19(31.6) & & \\
\hline \multicolumn{2}{|c|}{ Grading of Lymphoedema } & \multicolumn{2}{|c|}{ Daily washing of affected body area } \\
\hline GRADE 1 & $93(52.2)$ & Yes & $175(98.3 \%)$ \\
\hline GRADE 2 & $55(30.9)$ & No & $3(1.7 \%)$ \\
\hline GRADE 3 & 23(12.9) & & \\
\hline Didn’t had any edema & $7(3.9)$ & & \\
\hline \multicolumn{2}{|c|}{ Time of washing their affected part } & Practices of using customized footwear & $\mathrm{n}=152$ \\
\hline Morning time & $51(2.7 \%)$ & Yes & $148(97.4)$ \\
\hline Both morning and evening time & $30(16.7 \%)$ & No & $4(2.6)$ \\
\hline No specific time & $21(11.8 \%)$ & & \\
\hline $\begin{array}{l}\text { Both morning and night before } \\
\text { going to sleep }\end{array}$ & $76(42.7 \%)$ & & \\
\hline
\end{tabular}

viewed, 152 had lymphoedema of leg. Daily leg exercise is necessary for the morbidity management as recommended by the NVBDCP program. It was found that only $30 \%$ of them practiced one or the other exercise regularly (Figure 1). Elevation of the leg was practiced by $45 \%$ patients, 'Up on toe' exercise by $30 \%$, 'Toe pointing exercise' by $31 \%$, 'circle exercise' by $25 \%$. Wearing of customized footwear was practiced by only $2.6 \%$ (Table 3 ). Attendance to morbidity management camp was very less i.e $28 \%$. For protection against mosquitoes, $48 \%$ used mosquito repellent coils and only $6 \%$ used bed nets (Figure 2 ).

\section{DISCUSSION}

Global Programme to Eliminate LF (GPELF) focuses on two major components 1 . Interrupting transmission, 2. Managing morbidity and preventing disability. ${ }^{2}$ Present study focused on the second major component of the GPELF. One of the study conducted in tertiary care centre Kerala, ${ }^{11} 72.6 \%(82)$ had in the age group of $60-85$ years which was almost similar to our study were most of the patients were above age of 56 years $60.7 \%$ (108). Other similar study conducted in Srilanka ${ }^{12}$ had male patients $33.3 \%$ (22) and female patients $66.7 \%$ (44), both legs affected patients were 9\% (6). 45.5\% (30) were in Grade 1 stage and $16.7 \%$ (11) were in Grade 3 stage, which was similar to our study as female patients were $66.3 \%$ (118) and male patients $33.7 \%$ (60). Both legs patients were $21(11.8 \%), 52.2 \%$ (93) were in Grade 1 stage and $12.9 \%$ (23) were in Grade 3 stage. Knowledge of nail hygiene was lower in present study (57.3\%) compared to the above study in which it was above $80 \%$. A study conducted in northeastern Brazil ${ }^{13}$ showed that the patients who were not using bed nets at night were at two times high risk of developing infection than those who were using the bed nets at night $(\mathrm{OR}=1.87$; 95CI:1.47-2.38). As present study did not focus on the risk factors, rather use of protective measures was studied. Only $6.2 \%$ of the patients used bed nets and $4.5 \%$ used both bed nets and coils, $41 \%$ did not use any form of protective measure against mosquitoes.

\section{CONCLUSION}

The program has to ensure that all the LF patients will undergo morbidity management training and know how to take care of lymphoedema. A total of 178 people were interviewed in the study among them most of the patients i.e. $60.7 \%$ were above 55 years and women were more $(66.3 \%)$ than men $(33.7 \%)$. The literacy rate among subjects was fairly good, $31 \%$ had up to primary level $27 \%$ of them up to secondary level. Regarding knowledge on how filariasis is caused, women (32\%) had better knowledge than men (22\%). Regarding MDA both men and women had good knowledge and $95 \%$ of men and $84 \%$ women had received drugs during recent MDA. The lymphoedema of leg was found both in men and women. More than $50 \%$ of them had grade I stage lymphoedema followed by grade II (31\%). In morbidity management, washing was practiced by $98.3 \%$ among which majority $(42.7 \%)$ said that they washed both morning and night before going to sleep. Recommended exercises for morbidity management were not practiced by most of the patients.

\section{Limitations}

Due to the cross sectional study design, a temporal association cannot be made. Moreover, lesser duration of the study and in depth perception of the patients regarding morbidity management was not possible.

\section{ACKNOWLEDGEMENT}

I am greatly indebted to all Medical officers (PHCs/CHCs/UHCs) ASHA workers and Male health workers of concerned PHCs/CHCs/UHCs of Udupi District for sharing their valuable time and helping me for facilitating the data collection process at the field level.

\section{CONFLICT OF INTEREST}

None

\section{ABBREVIATIONS USED}

GPELF: Global Programme to Eliminate LF; LF: Lymphatic Filariasis; MDA: Mass Drug Administration; NVBDCP: National Vector Borne Disease Control Programme; NTD: Neglected Tropical Diseases; WHO: World Health Organization.

\section{REFERENCES}

1. Savioli L, Daumerie D. Sustaining the drive to overcome the global impact of neglected tropical diseases: second WHO report on neglected tropical diseases. 1st ed. Geneva. WHO press; 2013

2. Lymphatic filariasis programme [Internet] "The Global Programme to Eliminate Lymphatic Filariasis". [cited 2012 Oct 15]. Available from: http://www.who.int/ lymphatic_filariasis/disease/en/

3. Eric et al [Internet]. Programme to Eliminate Lymphatic Filariasis "MDA Progress". [cited 2012 Oct 15]. Available from: http://www.who.int/lymphatic_filariasis/disease/programme_progress/en/index.html

4. Weekly epidemiological record [Internet]. World Health Organization. No. 37, 2012, 87, 345-356. [cited 2012 Oct 17]. Available from: http://www.who.int/wer

5. Filariasis Control in India and its Elimination. National Vector Borne Disease Control Programme. Chapter 1: p 6-7. [cited 2012 Oct 17]. Available from: http:// nvbdcp.gov.in/filariasis.htm

6. Microfilaria of Wuchereria bancrofti and Brugia malayi (CDC Photo; DPDx) [In- 
ternet]. [cited 2012 Oct 19]. Available from: http://www.cdc.gov/parasites/lymphaticfilariasis/epi.html.

7. Clinical Manifestations of lymphatic filariasis [Internet]. [cited 2012 Oct 15]. Available from: http://www.who.int/lymphatic_filariasis/epidemiology/epidemiology_manifestations/en/index.html.

8. Chronic manifestations of lymphatic filariasis [Internet]. [cited 2012 Oct 15]. Available from: http://www. filariasis.org/disease.html\#asymptomaticpresentations

9. Lymphatic filariasis: Country indicators [Internet]. World Health Organization. [cited 2012 Oct 19]. Available from: http://www.who.int/neglected_diseases/ preventive_chemotherapy/lf/db/index.html?units=minimal\&region=all\&country $=$ ind \& countries $=$ ind \&year $=2011$.

10. Morbidity Management among lymphatic filariasis patients [Internet]. [cited
2012 Nov 03]. Available form: http://nvbdcp.gov.in/filariasis-new.html.

11. Sushma K, Aswathy S, Sanjeev S, Paniker N, Kavita D, Shamsul K. Morbidity trends in Lymphatic Filariasis: Analysis from tertiary care centre in Kerala, Southern India. G J Med Ph. 2012;1(2).

12. Nilmini TGAC, Ranjan P, Silva DN. Lymphoedema management knowledge and practices among patients attending filariasis morbidity control clinics in Gampaha District, Sri Lanka. Filaria journal 2004, 3:6. Available from: http:// www.filariajournal.com/content/3/1/6; https://doi.org/10.1186/1475-2883-3-6 ; PMid:15287989 PMCid:PMC514715.

13. Fatima A, Mauro M, Ricardo X, Cintia B, Silva MM, Andre F. Bancroftian Filariasis in two urban areas of Recife, Brasil: The role of individual risk factors. Rev. Inst Med Trop. 1995;37(3):225-33.

Cite this article : Basavaraj K, Bharatesh SK, Murali D, Ramachandra K, Sowmya M. A Study on Morbidity Management among Lymphatic Filariasis Patients in Udupi district, Karnataka, India. Int J Med. Public Health. 2017; 7(2):92-6. 